\title{
Effect of prolonged stimulation on cerebral hemodynamic: A time-resolved fNIRS study
}

\author{
Michele Butti \\ Dipartimento di Bioingegneria, IIT Unit, Politecnico di Milano, Piazza Leonardo da Vinci 32, \\ I-20133 Milan, Italy \\ Davide Contini \\ Dipartimento di Fisica, Politecnico di Milano, Piazza Leonardo da Vinci 32, I-20133 Milan, Italy \\ Erika Molteni \\ Dipartimento di Bioingegneria, IIT Unit, Politecnico di Milano, Piazza Leonardo da Vinci 32, \\ I-20133 Milan, Italy \\ Matteo Caffini \\ Dipartimento di Fisica, Politecnico di Milano, Piazza Leonardo da Vinci 32, I-20133 Milan, Italy \\ Lorenzo Spinelli \\ National Laboratory for Ultrafast and Ultraintense Optical Science, ULTRAS-INFM-CNR, Piazza \\ Leonardo da Vinci 32, I-20133 Milan, Italy
}

Giuseppe Baselli, Anna Maria Bianchi, ${ }^{\text {a) }}$ and Sergio Cerutti

Dipartimento di Bioingegneria, IIT Unit, Politecnico di Milano, Piazza Leonardo da Vinci 32, I-20133 Milan, Italy

Rinaldo Cubeddu

Dipartimento di Fisica, Politecnico di Milano, Piazza Leonardo da Vinci 32, I-20133 Milan, Italy; National Laboratory for Ultrafast and Ultraintense Optical Science, ULTRAS-INFM-CNR, Piazza Leonardo da Vinci 32, I-20133 Milan, Italy; Istituto di Fotonica e Nanotecnologie, IFN-CNR, Sezione di Milano, Piazza Leonardo da Vinci 32, I-20133 Milan, Italy; and Research Unit IIT, Politecnico di Milano, Piazza Leonardo da Vinci 32, I-20133 Milan, Italy

Alessandro Torricelli

Dipartimento di Fisica, Politecnico di Milano, Piazza Leonardo da Vinci 32, I-20133 Milan, Italy and Research Unit IIT, Politecnico di Milano, Piazza Leonardo da Vinci 32, I-20133 Milan, Italy

(Received 24 February 2009; revised 11 June 2009; accepted for publication 29 June 2009; published 12 August 2009)

Purpose: Sustained attention is one of the most important cognitive abilities for the management of everyday life, but it is often studied only at the behavioral level, while functional correlates are scarcely investigated. In this article, the authors address the topic of characterizing the dynamics of cerebral metabolism in the prefrontal cortex during a task of prolonged attention.

Method: By means of multichannel time-resolved functional near-infrared spectroscopy and generalized linear model based data processing, the authors measured the hemodynamic response of the prefrontal cortex from 19 healthy subjects to a shortened version of a sustained attention task (Conners' Continuous Performance Test), lasting for $10 \mathrm{~min}$.

Results: The task elicited significant brain activation, which did not remain constant for the entire task, but showed a drop not correlated with performance decay 4 min after the beginning of the task. Furthermore, oxygenated hemoglobin showed an increasing trend also during the first phase of the recovery, just after the end of the task.

Conclusion: The results indicate a nontrivial dynamics of neural activation, habituation processes, and hemodynamic/metabolic coupling. These results encourage further studies about continuous stimulation of cognitive functions on both healthy and pathological subjects. (C) 2009 American Association of Physicists in Medicine.

[DOI: 10.1118/1.3190557]

Key words: time-resolved fNIRS, sustained cerebral activation, hemodynamic changes, general linear model

\section{INTRODUCTION}

One of the most important goals of modern neuroimaging research is to understand the relationships between cortical task-induced activation and cerebral hemodynamic changes.
A great number of experimental protocols have been analyzed with functional techniques like functional magnetic resonance imaging (fMRI), positron emission tomography (PET), and functional near infrared spectroscopy (fNIRS) in order to find links between the two phenomena. The fNIRS 
technique allows monitoring temporal and spatial changes in oxygenated and deoxygenated hemoglobin concentrations $\left(\mathrm{O}_{2} \mathrm{Hb}\right.$ and $\mathrm{HHb}$, respectively). Continuous wave $(\mathrm{CW})$ fNIRS, however, is potentially biased by the hemodynamics in extracortical tissues (e.g., the variations in cutaneous blood flow). ${ }^{1,2}$ Time-resolved fNIRS overcomes these limitation and confounding effects, as it enables tissue depth discrimination based on time of flight of the scattered photons. ${ }^{3,4}$

Functional neuroimaging techniques provided us with the evidence of an early (2-3 s) increase in $\mathrm{HHb}$ and a simultaneous decrease in $\mathrm{O}_{2} \mathrm{Hb}$ in the hemodynamic response due to metabolic consumption, following cerebral activation during visual and motor tasks. ${ }^{5-7}$ Thereafter, the metabolic request is supplied by the organism with an increased contribution of blood flow and $\mathrm{O}_{2} \mathrm{Hb}$, exceeding the demand. ${ }^{8}$ Functional neuroimaging thus exploits these complex relationships both by assessing changes in cerebral blood flow $(\mathrm{CBF})$ and cerebral blood volume (CBV) and by capturing oxygenation-related signals. At the moment, fNIRS is the only technique potentially able to capture both responses given by $\mathrm{O}_{2} \mathrm{Hb}$ and $\mathrm{HHb}$ concentrations.

Most functional block design studies are based on short term (less than $1 \mathrm{~min}$ ) activation periods, usually interleaved by rest intervals and repeated in order to improve statistic significance, ${ }^{9-11}$ besides, the behavior of cerebral hemodynamic response during periods of prolonged (e.g., longer than 3 min) activation remains unclear, and little is known about long term adaptation of $\mathrm{CBV}$ and $\mathrm{O}_{2} \mathrm{Hb}$. Bandettini et $a l .{ }^{12}$ performed a visual task with fMRI recordings and observed high values of blood flow and oxygenation during the entire stimulation period $(20 \mathrm{~min})$. Similar results were obtained by Howseman et al. ${ }^{13}$ using different kinds of visual stimuli and various fMRI analysis techniques. Using fNIRS acquisitions and a visual task, Heekeren et al. ${ }^{14}$ found $\mathrm{HHb}$ values returning to basal level after $2 \mathrm{~min}$ of stimulation, whereas $\mathrm{O}_{2} \mathrm{Hb}$ values remained high during the whole task. Matsuda and Hiraki ${ }^{15}$ highlighted the fluctuations of $\mathrm{O}_{2} \mathrm{Hb}$ and $\mathrm{HHb}$ signal during the performance of a video game lasting several minutes.

Great uncertainty remains also in the characterization of the recovery period after stimulation. Some works attest a fast return to the baseline level, while others notice undershoots or overshoots with different amplitudes and latencies. ${ }^{12,16}$ Theoretical interpretations for these observations involve neurovascular uncoupling in the activated areas: Namely, an unbalance between oxygen consumption, regional $\mathrm{CBF}$, and regional $\mathrm{CBV}{ }^{17}$

Among all the cognitive faculties, which are deployed in everyday life, vigilance is one of the most frequently damaged in case of a traumatic event. It is known from literature that states characterized by a high level of vigilance mainly involve the activation of prefrontal and frontal regions. ${ }^{18,19}$ As neuropsychological tasks are a primary clinical investigation tool for the assessment of residual attentional skills in many neurological disorders and in post-traumatic patients, many of them are based on sustained/prolonged attentional tasks. Yet, very often these tests are solely evaluated by means of behavioral scores, while a simultaneous functional and hemodynamic assessment would support the clinical analysis and the patophysiological explanation of impaired attentional capabilities.

The purpose of this preliminary work is to address the topic of characterizing the dynamics of cerebral metabolism in the prefrontal cortex during a task of prolonged attention in a healthy population. At this purpose, measurements were performed on 19 healthy subjects, and time-resolved fNIRS data were analyzed by a generalized linear model (GLM) tailored to the specific protocol.

\section{MATERIALS AND METHODS}

\section{II.A. Time-resolved fNIRS}

A couple of pulsed diode lasers, operating at 690 and $820 \mathrm{~nm}$, with $80 \mathrm{MHz}$ repetition rate and $1 \mathrm{~mW}$ average power (PDL, Picoquant GmbH, Germany), are used as light sources. The laser heads are connected to multimode graded index fibers $(50 / 125 \mu \mathrm{m})$ by means of a homemade coupler that combines a neutral density attenuator (J54-082, Edmund OptiK $\mathrm{GmbH}$, Germany) with variable attenuation in the range of $0-80 \mathrm{~dB}$ and a standard FC fiber optics coupler. Multimode graded index optical fibers $(50 / 125 \mu \mathrm{m})$ with different lengths and a $2 \times 2$ fused fiber optic splitter (VISNIR5050, OZ Optics, Canada) are used to time multiplexing the laser pulses at the different wavelengths and to create two independent channels. In each channel a $1 \times 9$ fiber optic switch (F-SM19, PiezoJena GmbH, Germany) creates up to nine independent sources or injection points; therefore, 18 sources are available.

Four parallel detection chains accomplish acquisition of time-resolved reflectance curves. Each chain consists of a compact 4-channel photomultiplier tube with a multialkali surface (R5900-20-M4, Hamamatsu Photonics, Japan), a routing electronics (HRT-41, Becker\&Hickl, Germany), a fast amplifier (HFAC-26, Becker\&Hickl, Germany) and a PC board (SPC130, Becker\&Hickl, Germany) for timecorrelated single photon counting (TCSPC). The parallel use of the four detection and acquisition lines enables a total of 16 independent detectors. Fiber optic bundles, $1.5 \mathrm{~m}$ in length with a distal end $90^{\circ}$ bended and NA $=0.5$ (LLR 3/1500-SIK- $90^{\circ}$, Loptek GmbH, Germany), are used for light collection from tissue.

The system is controlled by a personal computer (Pentium IV $3.5 \mathrm{GHz}, 2$ Gbytes RAM), which hosts the acquisition boards and it is used for data storage. A homemade software, written in C language in the LabWindows/CVI environment (National Instruments, TX), is used to control the instrument. The software is interfaced to a microcontroller unit (dsPIC30F6014, Microchip Technology, Inc., AZ) which is used for the hardware control of the instrumentation. In particular, the microcontroller unit generates trigger signals for the synchronization of data acquisition by the TCSPC boards and of the fiber optic switches. Typically, by means of the microcontroller unit, the sources can be activated sequentially in any desired sequence. The instrument response func- 


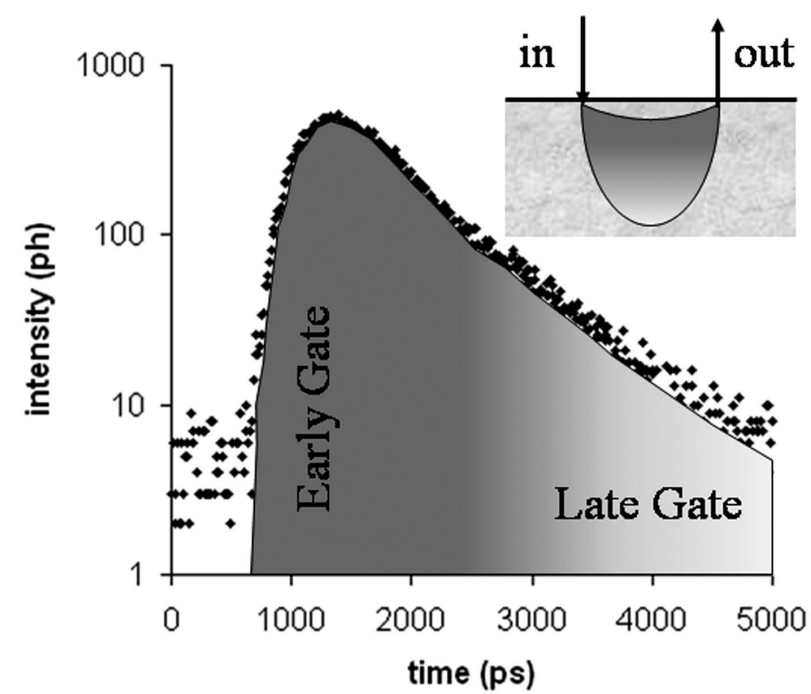

FIG. 1. Typical time-resolved fNIRS curve. The inset shows a schematic of the fNIRS measurement and of the visited regions by early (dark) and late (light) photons.

tion obtained by filling all the propagating modes of the bundle has a FWHM of approximately 500 ps. A detailed description and characterization of the system can be found in Contini et al. ${ }^{20}$

The system is also interfaced with dedicated software (Presentation, Neurobehavioral Systems, Inc., Albany, CA) for precise stimulus delivery and experimental control program.

For each wavelength $\lambda$ a reference time-resolved fNIRS curve $R_{0}(t ; \lambda)$ is derived by averaging the curves corresponding to the initial baseline period. Fitting of $R_{0}(t ; \lambda)$ yields the reference absorption value $\mu_{a 0}(\lambda)$. Then, at each recording time $T$ during the experiment, changes in the absorption coefficient are derived ${ }^{21}$ as

$$
\Delta \mu_{a}(\lambda ; T)=-\frac{1}{\nu t} \ln \left(\frac{R(t ; \lambda ; T)}{R_{0}(t ; \lambda)}\right),
$$

where $\nu$ is the speed of light in the medium, $t$ is the arrival time of photons, and $R(t, \lambda, T)$ is the time-resolved fNIRS curve at the recording time $T$. To enhance the contribution from deep layers and to remove possible disturbances caused by superficial layers, a correction method based on the use of late time windows $(t=1750-2500 \mathrm{ps})$ was also applied. ${ }^{22}$ It is well known that depth information in time-resolved fNIRS is encoded in the time of flight of photons: ${ }^{23,24}$ Early photons probe the superficial layers of the head, while late photons have a higher probability to visit deeper layers (see Fig. 1). Finally, the absorption coefficient is then derived as $\mu_{a}(\lambda ; T)=\mu_{a 0}(\lambda)+\Delta \mu_{a}(\lambda ; T)$. Taking the assumption that $\mathrm{O}_{2} \mathrm{Hb}$ and $\mathrm{HHb}$ are the main chromophores contributing to absorption, their concentrations are then derived by Lambert-Beer law. Then, the total hemoglobin content $\left(\mathrm{tHb}=\mathrm{HHb}+\mathrm{O}_{2} \mathrm{Hb}\right)$ and tissue oxygen saturation $\left(\mathrm{SO}_{2}\right.$ $\left.=\mathrm{O}_{2} \mathrm{Hb} / \mathrm{tHb}\right)$ are calculated.

\section{II.B. Generalized linear model}

The fNIRS time series were analyzed with the generalized linear model, which is a well known statistical method suitable for analyzing time series data. ${ }^{25}$ The GLM is regularly applied in fMRI data analysis. ${ }^{26-28}$ A time series $\left(\mathrm{O}_{2} \mathrm{Hb}\right.$ or $\mathrm{HHb}$ time course) for a given channel is modeled as a linear combination of $L$ regressors (known functions) plus an error term,

$$
\underset{(T \times 1)}{Y}=\underset{(T \times L)(L \times 1)}{X} \underset{(T \times 1)}{\beta},
$$

where $X$ is called the design matrix $(T \times L$ matrix $)$, each column is a regressor, and $T$ is the number of time points. $\beta$ is the vector of the unknown parameters, one for each regressor, weighing their importance in modeling the original signal. Errors $\varepsilon$ are assumed to have normal distribution with zero mean and covariance $\sigma^{2} V$,

$$
\stackrel{i i d}{\varepsilon \approx N_{J}\left(0, \sigma^{2} V\right) .}
$$

The origin for correlation on error terms can be found in cardiac, respiratory, and vasomotor effects. The ordinary least squares estimation of the parameters is

$$
\hat{\beta}=\left(X^{T} X\right)^{-1} X^{T} Y .
$$

Estimation of the error covariance component is performed in two steps; first the correlation matrix $V$ is evaluated using the restricted maximum likelihood procedure ${ }^{29}$ and then the variance is calculated, for each time series, using the estimation

$$
\hat{\sigma}^{2}=\frac{\hat{e}^{T} \hat{e}}{\operatorname{tr}(R V)},
$$

with $R$ as the residual forming matrix. Hypotheses on predictor variables can be tested constructing the statistic

$$
F=\frac{\left(\hat{\beta}_{i} / \sqrt{\operatorname{var}\left(\hat{\beta}_{i}\right)}\right)^{2}}{L} .
$$

This value is a Fisher distribution $F_{L, \psi}$, with $\psi$ $=\operatorname{tr}(R V)^{2} / \operatorname{tr}(R V R V)$ (Satterthwaite approximation, where $\psi$ is the approximation and $R V$ is the variance). The interest of this work was the analysis of the mean level of brain activation, deducible from levels of $\mathrm{O}_{2} \mathrm{Hb}$ and $\mathrm{HHb}$, and of its time course. Consequently, boxcar regressors marking with a unit value subsequent time windows and null outside were chosen. The duration of this epochs was empirically set to $2 \mathrm{~min}$ according to the trade-off between time resolution and statistical significance. An example of the applied signal modeling is showed in Fig. 2. The GLM was applied separately for $\mathrm{O}_{2} \mathrm{Hb}$ and for $\mathrm{HHb}$ data. Cerebral activation is indicated by positive $F$ values in the $\mathrm{O}_{2} \mathrm{Hb}$ time series and by negative $F$ values for $\mathrm{HHb}$.

Inferential procedures at the group level (e.g., about activated channels over all subjects) can be performed using the fixed-effect approach. The above formalism was applied to 


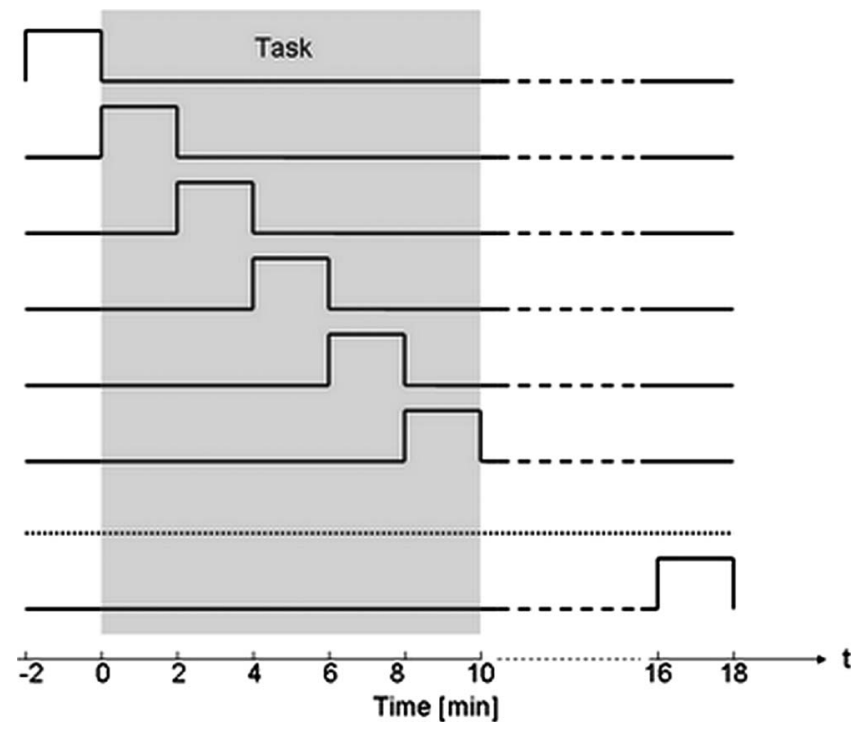

FIG. 2. Regressors for the metabolic fNIRS data. The regressors constitute the design matrix for the experiment. Each regressor models the effects of activation over $2 \mathrm{~min}$.

data from all subjects sequentially concatenated forming a group data vector. The design matrix was accordingly modified.

For each subject and for each fNIRS channel, we calculated the Pearson product moment correlation coefficient of the residuals of beta estimation at lag 1 . The results confirmed that residuals were not significantly different from zero for both oxygenation and deoxygenation series if the $P$ value was set at 0.05 . Then, no corrections for serial correlations were applied.

\section{II.C. Subjects}

Nineteen healthy volunteers, comparable for age and school attendance participated in the experiment. All subjects were males, right handed, with a mean age of $29 \mathrm{yr}$ (SD of $5 \mathrm{yr}$, range of 22-41 yr). All volunteers were native Italian speakers and were not paid to participate. The neuropsychological status of each participant was assessed by means of an unpublished adapted italian version of the "Neuropsychological History Questionnaire. ${ }^{, 30}$ They all had normal vision and had no history of psychiatric disorders. None of them showed any neuropsychological illness, cognitive level and attentive capability were normal, and none of them had any first-degree relatives with a psychiatric illness. The study received the approval of the local Ethics Committee. Moreover, written informed consent was obtained from all volunteers after the examination and test procedure had been explained.

\section{II.D. Activation task}

The present study is based on the "not- $X$ " version of the continuous performance test (CPT), often called the Conners' CPT. ${ }^{31}$ Different letters were randomly presented in the central portion of a computer screen. The subject was sitting

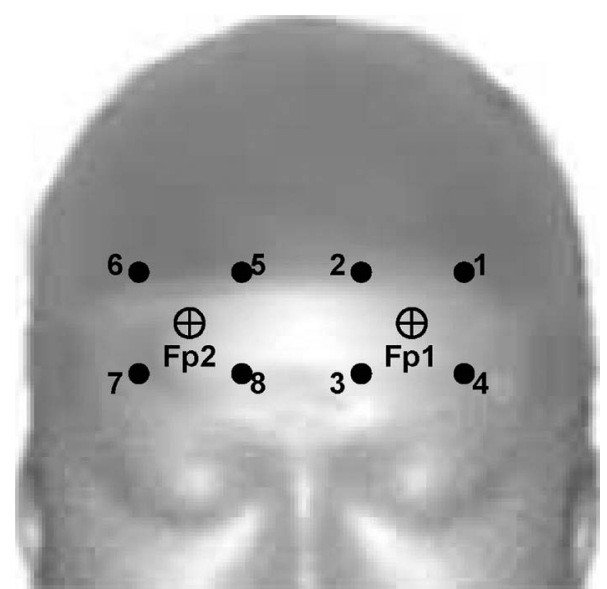

FIG. 3. Position of probes over the frontal head. Two emitters were placed according to $\mathrm{Fp} 1$ and $\mathrm{Fp} 2$ positions of 10/20 standard EEG system (black crosses); eight receivers were placed around the two emitters at a constant distance (dots with numbers from 1 to 8 ).

in front of the screen. He had to click the left mouse button as fast as he could whenever a letter appeared and not to press any key at the appearance of the target letter X. Instructions were given to all subjects before the test. The attention task was $10 \mathrm{~min}$ long. Letters were presented with interstimulus interval of 1 or $2 \mathrm{~s}$ and remained on the screen for $250 \mathrm{~ms}$ (stimulus duration). A total of 450 letters was presented, with probability of critical stimulus set at $6 \%$. An initial baseline lasting $2 \mathrm{~min}$ and a final recovery period lasting $8 \mathrm{~min}$ were also recorded for a total protocol duration of $20 \mathrm{~min}$. During baseline and recovery, the subjects were presented a meaningless image (differently oriented geometrical lines) to which no response was required.

Scientific literature provides several nomenclatures for events and responses. ${ }^{32,33}$ In this paper, letter $\mathrm{X}$ was regarded as the target or No-Go event and also as the infrequent stimulus. On the contrary, any letter other than $\mathrm{X}$ was regarded as the nontarget, alias Go event or frequent stimulus. Any correct reaction to the target stimulus $\mathrm{X}$ was a correct rejection; any incorrect answer to the target was a commission error, alias false alarm. Any correct reaction to the nontarget stimuli (letters other than $\mathrm{X}$ ) was a correct response; any incorrect answer to the same nontarget was an omission error and can be regarded as a missing response. Hits, i.e., the total number of correctly and incorrectly given answers, were not considered in this work due to the poor specificity of such an index.

\section{II.E. Optodes localization}

The optical probe was placed over the head in order to cover the underlying prefrontal cortex and light sources were centered (according to the international 10-20 system for the EEG electrode placement) at the Fp1 and Fp2 for left and right sides, respectively (Fig. 3). The source-detector distance was $2 \mathrm{~cm}$.

In CW NIRS measurement depth is strictly dependent on the source-detector distance. Therefore, it is common to use 
a large source-detector distance (of the order of $3-4 \mathrm{~cm}$ ) to probe the brain cortex, on average, $1-3 \mathrm{~cm}$ below the scalp, depending on the position over the head. ${ }^{34}$ Moreover, measurement depth for CW NIRS depends also on both the absorption and the scattering coefficient of the sample. Conversely, in time-resolved NIRS measurement depth is dependent on the time of flight of reemitted photons and on the scattering properties of the sampled medium, but it is not affected by the source-detector distance and by the absorption coefficient of the sample. ${ }^{24}$

The source-detector distance of $2 \mathrm{~cm}$ used in this study is a value that already proved to be sensitive to hemodynamic changes in the adult brain cortex. ${ }^{20}$ Indeed, we have recently verified that time domain NIRS measurements at null or small (i.e., $0.2 \mathrm{~cm}$ ) source-detector distance can be used to monitor hemodynamic changes in the adult brain cortex. ${ }^{35,36}$

No downward sliding of the fiber holders was observed at the end of the measurements in any subject. Custom-made fiber holders were designed to keep fibers normally to the forehead by black rubber pads. Holders were kept in place on the head of the subject by biadhesive tape and velcro bands. The minimum distance between the light source located on the left (right) forehead and the detectors placed over the right (left) forehead was $5 \mathrm{~cm}$ in order to avoid cross-talk between optical signals detected simultaneously in the two areas.

\section{II.F. Statistical analysis}

To examine whether there were statistically significant changes in fNIRS parameters, amplitude analysis on the $\mathrm{O}_{2} \mathrm{Hb}$ and $\mathrm{HHb}$ was performed. All repeated measures ANOVAs had two within-group factors: Condition (baseline vs task vs recovery) and region (upper left-Chn 1, Chn 2; lower left-Chn 3, Chn 4; upper right-Chn 5, Chn 6; lower right-Chn 7, Chn 8). Post hoc analyses were performed with pairwise ANOVAs. A $P$ value of 0.05 was considered to be statistically significant. The Greenhouse-Geisser procedure was used to correct for sphericity violations, where necessary.

All the statistical analyses were performed with NCSS statistical software (NCSS Statistical \& Power Analysis Software, http://www.ncss.com).

\section{RESULTS}

\section{III.A. Behavioral data}

The not-X CPT performance data for the subjects were as follows: Reaction time for frequent stimuli of $310.2 \mathrm{~ms}$ (SD: $38.6 \mathrm{~ms}$ ), reaction time for errors of $271.3 \mathrm{~ms}$ (SD: $30.9 \mathrm{~ms}$ ), and number of errors of 12.2 (SD: 4.9). Comparison with literature evidenced that the subjects provided acceptable results even though they performed slightly worse than healthy people usually do. Nevertheless, the reader should consider the "nonstandard" setting in which the test was conducted: Subjects had to perform the task in a completely dark room in order to optimize the operating conditions of fNIRS device, and they had to wear laser fibers and a probe over their head in the meanwhile. These factors may have contributed to worsen a little the behavioral results, with respect to what is generally reported.

A statistically significant difference was found on reaction time for frequent stimuli and errors $(p<0.01)$. Reaction times for errors and frequent stimuli and the number of errors were not statistically different along time $(p>0.05)$.

Reaction time for the frequent stimulus and the number of errors showed a negative linear correlation by Pearson's coefficient $(r=-0.57, p<0.05)$. No correlation between fNIRS parameters (difference of activity between task and baseline) and the behavioral data (reaction time for correct response and commission errors, number of errors) was found.

\section{III.B. Time-resolved fNIRS}

The not-X CPT attentional test significantly altered the presence of $\mathrm{O}_{2} \mathrm{Hb}$ and $\mathrm{HHb}$ in the prefrontal cortex of the analyzed population. As an example, $\mathrm{O}_{2} \mathrm{Hb}$ (light line) and $\mathrm{HHb}$ (dark line) time courses for a single subject (raw data) during the entire experiment time are shown in Fig. 4. Active task lasts from second 120 to second 720 . The task caused an increase in $\mathrm{O}_{2} \mathrm{Hb}$ associated with a decrease in $\mathrm{HHb}$, thus suggesting that the anterior attentional system activates. Interestingly, the fNIRS parameters modified by the task did not seem to return to the initial baseline level during the recovery phase, after the end of the stimulation.

On the other hand, the relatively large variability over the sensors (put into evidence in Fig. 4 as well) should also be taken into consideration. Indeed, the advantage that timeresolved fNIRS technique allows some discrimination among cerebral layers and, consequently, a better spatial resolution, is counterbalanced by the presence of a higher level of noise due to the smaller number of photons captured. In this light, combined use of time-resolved fNIRS and advanced statistical data analysis is encouraged, being quite approximate a simple visual analysis of the data (often presented in studies with continuous wave NIRS).

Repeated measures ANOVA revealed a significant main effect of condition $(p<0.05$ for $\mathrm{HHb}$ and $p<0.01$ for $\mathrm{O}_{2} \mathrm{Hb}$ ), while no reliable main effect of region $(p<0.1)$ or condition-by-region interaction $(p<0.5)$ was found.

Pairwise post hoc comparisons were statistically significant in the following tests: $\mathrm{HHb}$, baseline vs task ( $p$ $<0.001)$ and task vs recovery $(p<0.05), \mathrm{O}_{2} \mathrm{Hb}$, baseline vs task $(p<0.01)$, and baseline vs recovery $(p<0.01)$. Averaged values over subjects and channels are presented in Fig. 5.

Grand averaged values of $\mathrm{O}_{2} \mathrm{Hb}$ and $\mathrm{HHb}$ are reported in Fig. 6 in order to better enlighten the temporal dynamics of the acquired parameters. They are plotted by channels over a time window of $2 \mathrm{~min}$. As already pointed out, both $\mathrm{O}_{2} \mathrm{Hb}$ and $\mathrm{HHb}$ are affected by the task, exhibiting maximum of variation approximately in the middle of the task period. After that, $\mathrm{HHb}$ gradually returns to baseline value during the remaining part of the stimulation and the following recovery. $\mathrm{O}_{2} \mathrm{Hb}$, conversely, shows a steeper decrease during the sec- 

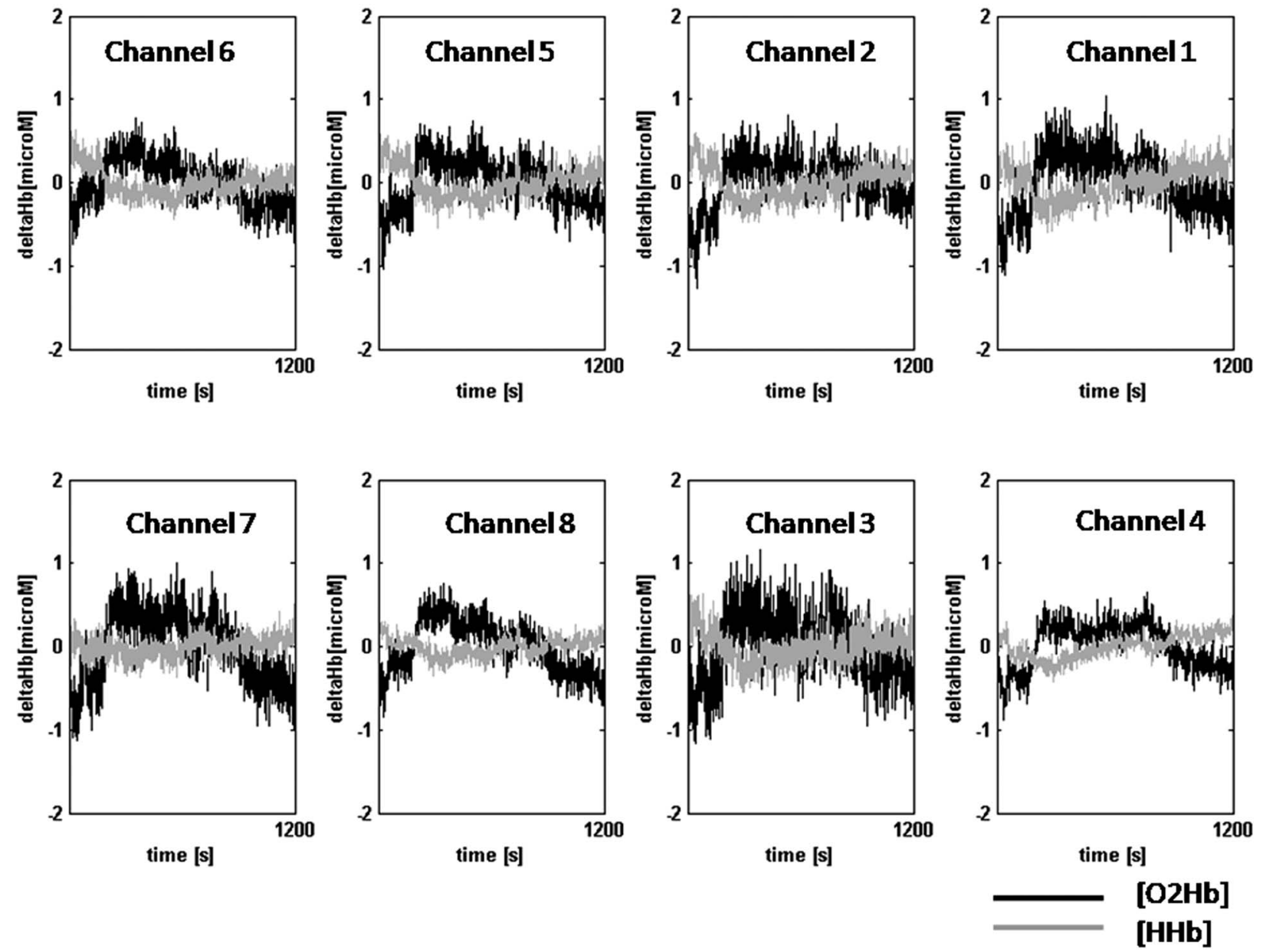

FIG. 4. Changes in $\mathrm{O}_{2} \mathrm{Hb}$ (light line) and $\mathrm{HHb}$ (dark line) for one subject and all channels during the not-X CPT task. Active task lasts from second 120 to second 720 .

ond part of the task, followed by a new increase at the end of the stimulation. This trend is manifested in all channels even though with different ranges of variation.

To investigate more deeply and with more statistical detail the spatiotemporal evolution of the hemodynamic response following the not-X CPT task, fNIRS data were then analyzed by means of the GLM theory. The time course was modeled as a series of consecutive boxcars in order not to force the response into a staircase shape with a 2 min reso-
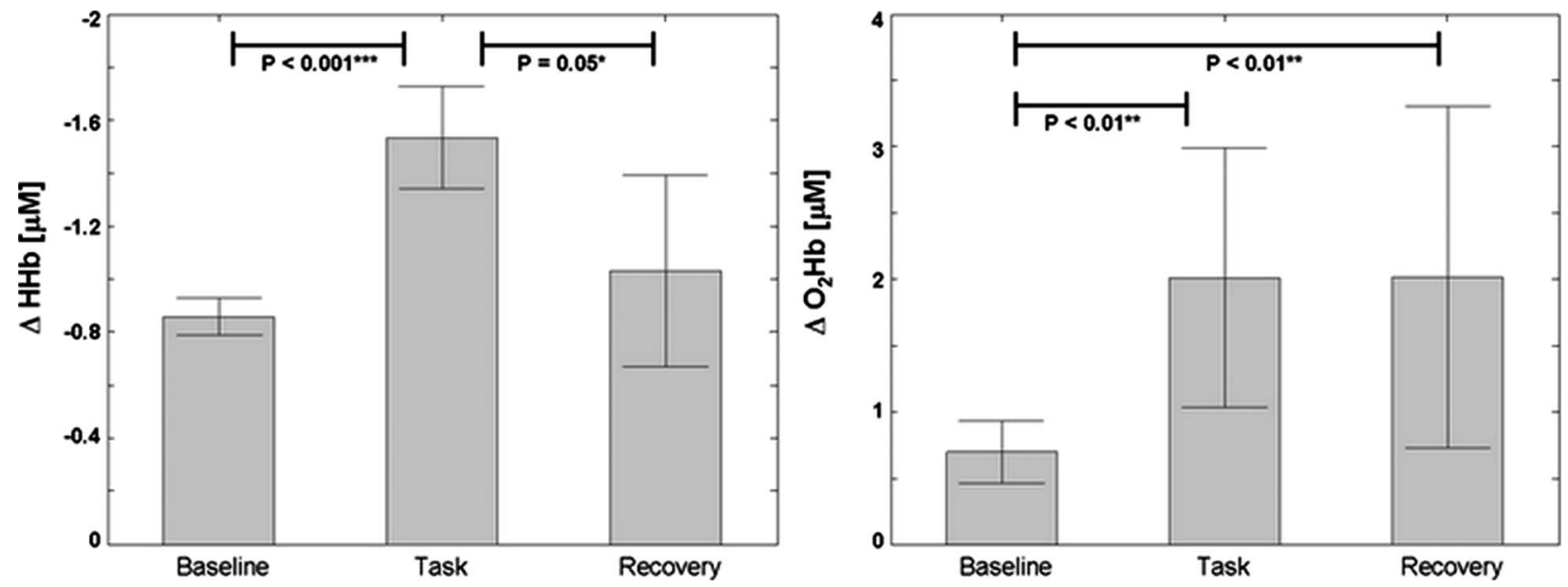

FIG. 5. $\mathrm{HHb}$ (left panel) and $\mathrm{O}_{2} \mathrm{Hb}$ (right panel) concentration changes during baseline, task, and recovery. Gray bars are mean values. Changes are estimated with respect to the initial $20 \mathrm{~s}$ of the baseline. 

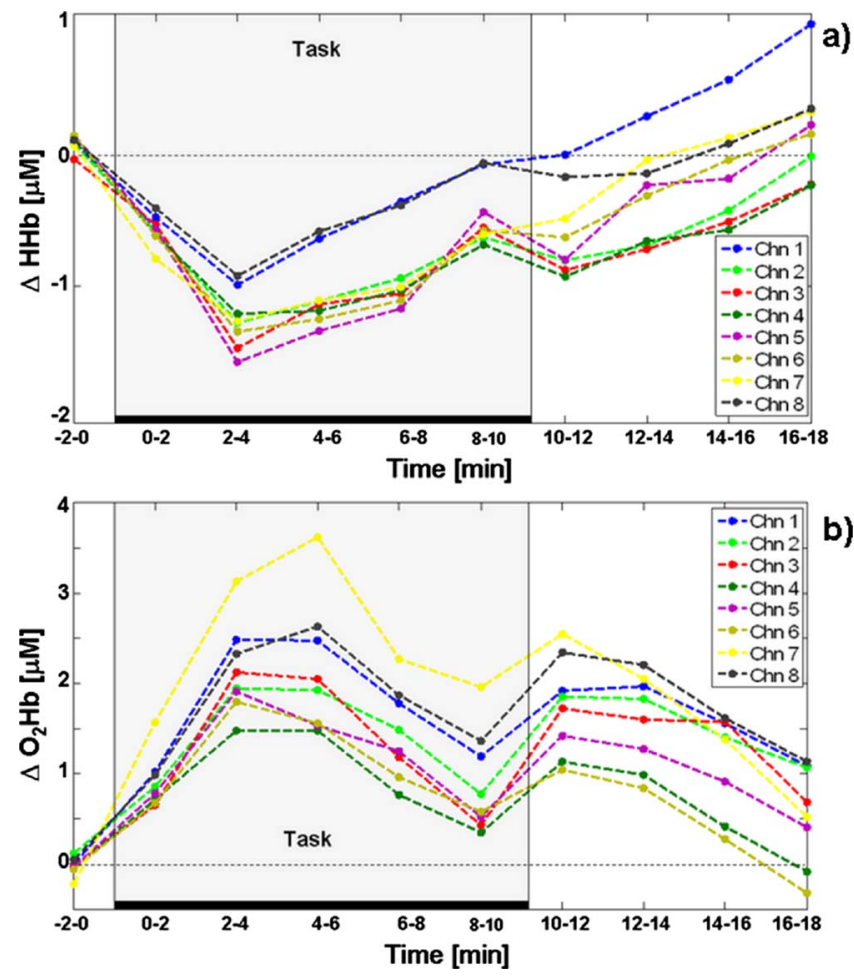

FIG. 6. Averaged values over subjects and over time windows of 2 min for the hemodynamic parameters. Cerebral activation is indicated by a $\mathrm{HHb}$ decrease and an $\mathrm{O}_{2} \mathrm{Hb}$ increase. Values for all channels are presented. The results for $\mathrm{HHb}$ are shown in (a) and those for $\mathrm{O}_{2} \mathrm{Hb}$ are shown in (b).

lution. An example of the procedure is presented in Fig. 7 for one subject: The original time course, the estimated $\beta$ values of the system, and the error term are shown.

Statistical maps are generated from the values of the $F$-statistic, in its turn obtained from the resolution of the complete model at group level. As in fMRI analysis, the results have an straightforward statistical significance. Figure 8 shows the grand average activation maps, obtained from the values of the $F$-statistics. These $F$-maps refer to the prefrontal cortex, located under the probe. Different maps for $\mathrm{HHb}$ (left side of the image) and $\mathrm{O}_{2} \mathrm{Hb}$ (right side of the image) are presented.

$\mathrm{HHb}$ decreases with the task and reaches a minimum during 3-4 min of the test. This level is not maintained but after that it shows a slow increase for the remaining part of the stimulation period. This trend persists during the recovery time, without evident variations, regaining the initial values only at the end of it. Spatial distribution remains quite uniform along the test. A higher activity on the superior-frontal channels is observed compared to that recorded on the prefrontal area. $\mathrm{O}_{2} \mathrm{Hb}$ level increases, as expected, with the test; it reaches a plateau and maintains this level from 3 to $6 \mathrm{~min}$. In the remaining part of the task period, it shows a strong decrement, reaching almost the baseline level during the final $2 \mathrm{~min}$ of the test. As soon as the task ends, the $\mathrm{O}_{2} \mathrm{Hb}$ reveals a new and rapid increase, reaching a maximum in the first 2 min of recovery. During the following 4 min of recovery, a slow decrease in the concentration level is observed, reaching a basal level only at the end of the recording.
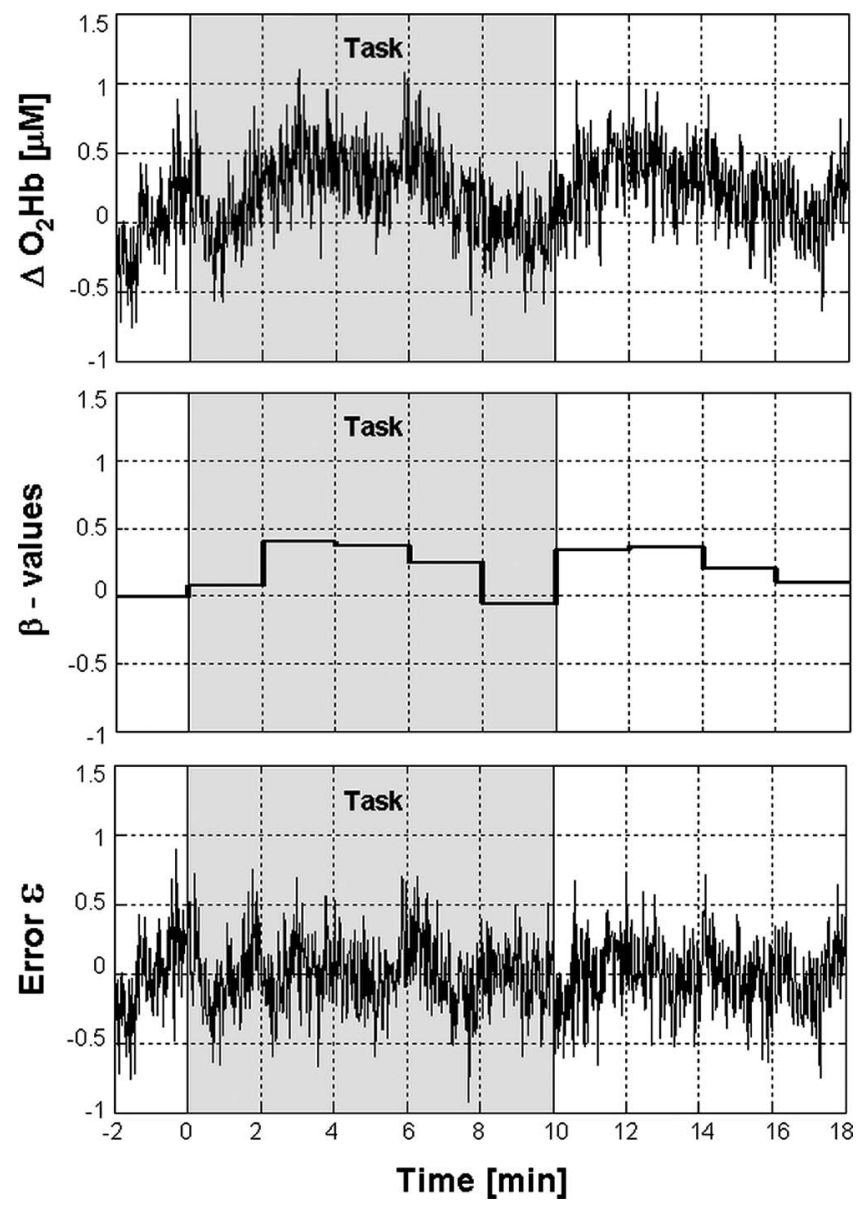

FIG. 7. Example of application of the GLM model to a $\mathrm{O}_{2} \mathrm{Hb}$ time course for one subject. The upper panel shows the original $\Delta \mathrm{O}_{2} \mathrm{Hb}$ time course. In the middle panel are presented the estimated $\beta$ values for the regressors, after resolution of the system. The lower panel presents the error term $\varepsilon$.

To verify how well the model approximates real data points, $R$-squared measures were computed. The $R$-square values, i.e., the proportion of the total variance explained by the model, were calculated on $\mathrm{O}_{2} \mathrm{Hb}$ and $\mathrm{HHb}$ data, separately for each channel. Results are displayed in Table I. As it can be seen, the chosen modelization presents a quite fair ability to predict the data.

The adequacy of the model was also checked out observing the residuals $\hat{\varepsilon}$. The error term is assumed to be a normal random variable with zero mean and covariance $\sigma^{2} V$. We assessed the normality of studentized residuals for each subject and each channel with Anderson-Darling test and then we performed tests on the hypotheses of mean $=0$ (t-test) and of variance $=1$ (chi-square test). All the tests performed confirmed the studentized residuals to be drawn from an $N(0,1)$ distribution at a significant level of 0.05 , thus confirming the fitting of the model.

A key feature of time-resolved fNIRS measurements is the possibility to assess absolute values of the hemodynamic parameters, thanks to the discrimination between absorption and scattering coefficients. Time-resolved fNIRS data acquired in the baseline period (before the performance of the Conners' CPT test) were analyzed. Figure 9 shows absolute 


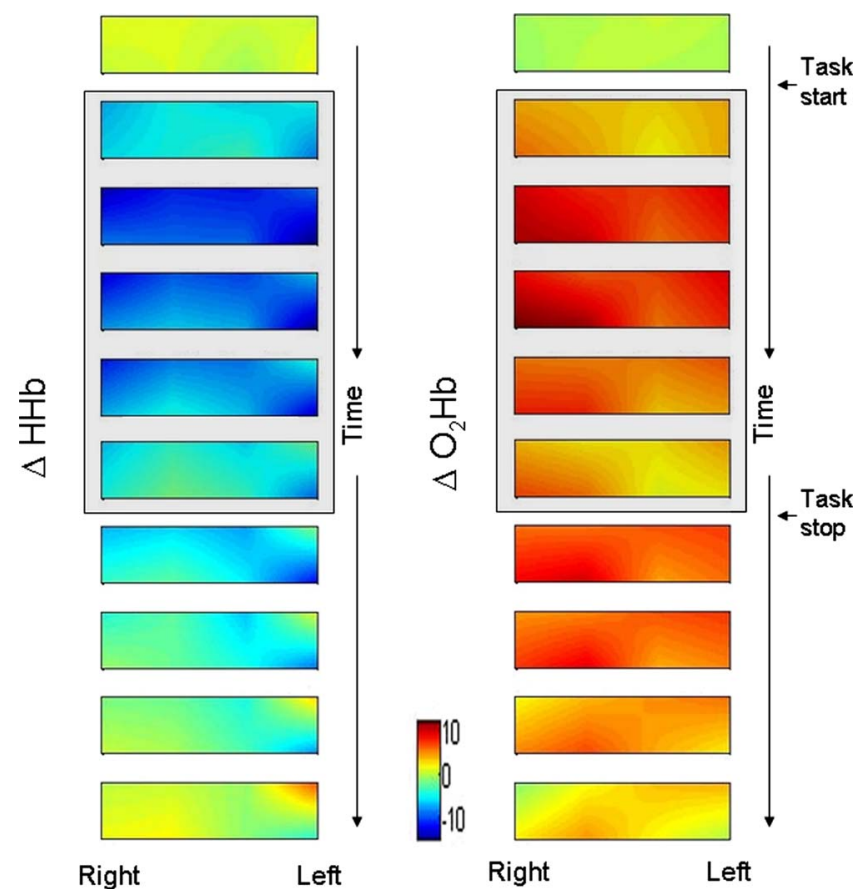

FIG. 8. Cortical activation during not-X CPT task detected by time-resolved fNIRS. The activation maps are expressed in terms of $F$-statistic, assessed by a second level analysis of the GLM model. Left column shows the HHb data and right column shows $\mathrm{O}_{2} \mathrm{Hb}$ data. Each map is relative to $2 \mathrm{~min}$ of acquisition for a total of 10 maps ( 2 min baseline -1 map, 10 min task-5 maps, 8 min recovery -4 maps).

values ( $\mu \mathrm{M}$ units) of cerebral $\mathrm{O}_{2} \mathrm{Hb}, \mathrm{HHb}$, and $\mathrm{tHb}$, together with $\mathrm{SO}_{2}$ percentage during baseline acquisition. No relevant differences were found either between hemispheres or among standard deviation values, which in all cases are kept small. These evidences proved measurement repeatability and convalidated data interpretation.

Movement artifacts might result in a sudden change in the measured scattering coefficient and in the variation in the detected light intensity, especially at early time, due to large reflection from superficial layers or from the surface. During data analysis we have inspected the data to check for any sudden change in the scattering coefficient. No significant occurrence was found. Moreover, as described in Sec. II, to enhance the contribution from deep layers and to remove possible disturbances caused by superficial layers, a correction method based on the use of late time windows $(t$ $=1750-2500 \mathrm{ps})$ was also applied. ${ }^{22}$

TABLE I. The $R$-square values of $\mathrm{O}_{2} \mathrm{Hb}$ and $\mathrm{HHb}$ data in each channnel.

\begin{tabular}{lcccc}
\hline \hline & Chn 6 & Chn 5 & Chn 2 & Chn1 \\
\hline $\mathrm{O}_{2} \mathrm{Hb}$ & 0.79 & 0.68 & 0.69 & 0.70 \\
$\mathrm{HHb}$ & 0.86 & 0.77 & 0.74 & 0.75 \\
& $\mathrm{Chn} 7$ & $\mathrm{Chn} 8$ & Chn 3 & Chn4 \\
\hline $\mathrm{O}_{2} \mathrm{Hb}$ & 0.66 & 0.76 & 0.59 & 0.77 \\
$\mathrm{HHb}$ & 0.76 & 0.77 & 0.63 & 0.82 \\
\hline \hline
\end{tabular}

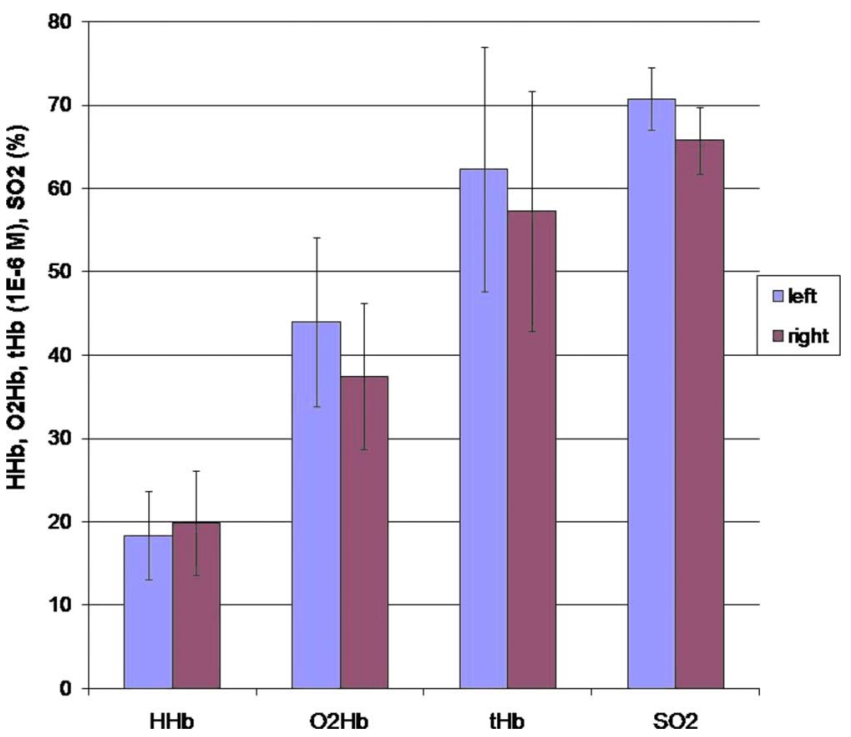

FIG. 9. Absolute values ( $\mu \mathrm{M}$ units) of cerebral $\mathrm{O}_{2} \mathrm{Hb}, \mathrm{HHb}, \mathrm{tHb}$, and $\mathrm{SO}_{2}$ percentage during baseline acquisition. Standard deviations are also shown. The constancy of values and standard deviations across hemispheres confirm the validity of measurements.

\section{DISCUSSION}

\section{IV.A. Discussion of methods}

fNIRS data analysis is often conducted reporting $\mathrm{O}_{2} \mathrm{Hb}$ and $\mathrm{HHb}$ concentrations recorded during the experimental conditions (e.g., test vs baseline) and comparing differences in metabolic values. ${ }^{37-39}$ Recently, a GLM approach was proposed to investigate spectroscopic data. ${ }^{40}$ The origin of this idea is the similitude of the information carried by the fNIRS and the fMRI on the cerebral metabolism, ${ }^{41,42}$ and in particular, the link between $\mathrm{HHb}$ and BOLD effects. ${ }^{43}$ GLMs are used in fMRI to detect cerebral region with neuronal activity. ${ }^{26-28}$ The use of this statistical method allows to overcome the absolute value of the data and to obtain a result with intrinsic statistical significance. Moreover, the group level analysis consists in a simple extension of the model used for single subject analysis. The first study proposing the use of GLM with fNIRS data is in Schroeter et al. ${ }^{40} \mathrm{~A}$ blocked design analysis, with baseline/task alternation, was applied, together with a visual stimulation protocol. This analysis is often used in fMRI ${ }^{44,45}$ but makes two hypotheses: stability of the response during each block of stimulation and same level of activation in the different activation periods. These hypotheses are not a priori verified in an experiment of prolonged stimulation aimed to characterize the parameter values during time. Later, Plichta et al. $^{46}$ applied GLM to a rapid event-related paradigm with visual stimulations of different contrast levels, giving a cortical mapping of activation in the visual area, for different experimental conditions; this experiment could assess modifications in the hemodynamic response following changes in stimulation, but not differences in perfusion due to more complex phenomena such as habituation and recoupling of the metabolic parameters. Very recently open source softwares have been proposed to analyze fNIRS data by a GLM 
approach. ${ }^{47-49}$ In our work we apply GLM to fNIRS data for the study of sustained activation. The flexibility of this statistical tool may be fully exploited in designing an analysis scheme able to follow parameter changes over time. A series of boxcar functions, each modeling a single activation period, has enough statistical power to partition a long lasting task in a bunch of consecutive segments. This modeling allows observing cerebral activation dynamics during cognitive activity of long duration.

\section{IV.B. Discussion of results}

In the present study, a modified version of the not-X CPT task was used. Not-X CPT test is commonly used in clinics for the assessment of response inhibition and sustained attention and was modified herein at the purpose of optimizing the hemodynamic stress in the brain. Sustained attention has been hypothesized to involve several prefrontal regions, especially the dorsolateral prefrontal cortex $;^{50-56}$ moreover, NIRS literature reports a decrease in $\mathrm{HHb}$ and an increase in $\mathrm{O}_{2} \mathrm{Hb}$ during the performance of several cognitive tests. ${ }^{57,58}$

Fallgatter and Strik $^{33}$ found a prevalent right-hemisphere frontal activation using a CPT test and a two-channel fNIRS acquisition. With multichannel fNIRS, Herrmann et al. ${ }^{59}$ documented cortical activation distributed over many channels for a "Go/No-Go" task, with no hemispheric differences, but the absence of lateralization is justified by missing statistical power due to small sample size. Our results indicated a prevailing right activity by $\mathrm{O}_{2} \mathrm{Hb}$ response, while $\mathrm{HHb}$ showed a less localized activity, distributed over the frontal lobe and with a slight prevalence on the left side. Casey et al. ${ }^{60}$ and Adler et al. ${ }^{61}$ reported distributed dorsolateral and orbitofrontal fMRI activation for response inhibition and a simple attention task, respectively. As concentration of $\mathrm{HHb}$ was demonstrated to partially determine the fMRI BOLD signal, $8,62-65$ our finding of a diffuse, fairly symmetrical $\mathrm{HHb}$ during a challenging cognitive task seems to be in agreement with the abovementioned fMRI studies.

Temporal trend of hemodynamic values during sustained activation was reported in many works. Bandettini et al. ${ }^{12}$ investigated flow and oxygenation level on prolonged stimulation by means of fMRI: A large number of experiments were performed, involving visual and motor stimulation and different MRI techniques. Almost all stimuli originated a rapid initial increase, followed by a steady-state elevated level for both flow and oxygenation. In a small number of subjects a decrease in those same parameters was observed after about 7-10 min of continuous stimulation and was related to neuronal habituation.

Different from what was reported in Bandettini et al., ${ }^{12}$ in our study fNIRS parameters did not show a fast level change. We observed a slow increase in activation during the first minutes of task, which reaches a maximum in about 4-5 min; after that, its value underwent a decrease until the end of the test. These results are in accordance with Matsuda and Hiraki ${ }^{15}$ and seem to provide further evidence that cerebral activity does not maintain the same level of activation for the overall period during complex sustained tasks.
Hathout et al. ${ }^{66}$ and Frahm et al. ${ }^{67}$ attested that the uncoupling between $\mathrm{CBF}$ and cerebral metabolic rate for oxygen $\left(\mathrm{CMRO}_{2}\right)$ is a phenomenon lasting about $2 / 4 \mathrm{~min}$; after that time, a new equilibrium between flow and oxidative metabolism is reached, and the coupling is restored. Therefore, the apparent reduction in activation after 4 min could be partially explained by a restored balance between hemodynamic response and metabolic demand.

In a PET study of continued activation on a visual task, Mintun et al. ${ }^{68}$ attested a regional CBF rising after 1 min of stimulation and presenting the tendency to last over time; conversely, the $\mathrm{CMRO}_{2}$ increase is small at the initial phases of the test and tripled toward the end (25 min). Hypothesizing a constant level of energy utilization, these authors inferred that the greatest part of the initial energy request reposes in the glycolysis. Further investigations will clarify this interpretation.

It is widely accepted in literature that the mechanisms of attention are characterized by more complex dynamics, if compared to pure sensory stimulation. ${ }^{69,70}$ Accordingly, our findings show that the rise time of activation is remarkable and is probably determined by a progressive involvement and focusing of the subject; moreover, this trend adds up to the instantaneous sensory contribution, clearly visible at the very beginning of the stimulation. After that, the situation of maximal activation is likely exceeding the need of the task and a decrease is detected, probably associated with the abovementioned recoupling between $\mathrm{CBF}$ and $\mathrm{CMRO}_{2}$. This tendency to the recoupling of hemodynamic parameters after prolonged stimulation is also attested in Hedera et al. ${ }^{71}$

Moreover, one of the phenomena most frequently encountered in the study of sustained attention is habituation: Against a gradual decrease in the neurohemodynamic activation, behavioral performance of the examinee remains stable. ${ }^{72,73}$ In other words, the brain progressively automates the carrying out of the task and gradually reduces the energetic request, without negatively affecting the performance.

Our results show accordance with this interpretation: Performance is not affected by repetitiveness and automatism of the stimulus/response pattern, while metabolic parameters indicate a reduction in energy consumption. After task cessation, a strong overshoot related to the $\mathrm{O}_{2} \mathrm{Hb}$ level was found in our data, starting abruptly and peaking after 2-3 min; a final return to baseline was then observed. $\mathrm{HHb}$ values marginally showed analogous behavior.

Literature results mainly regard the characteristics of single post-task periods of visual stimulation or motor task and document rapid return to baseline or small post-task undershoot. ${ }^{13}$ Conversely, in cognitive experiments, task periods are intermixed with rest periods; hence, compromising the possibility to observe effect of continuous activation on the post-task. ${ }^{74}$ Furthermore, the post-task is generally recorded for a very time-limited interval, thus precluding the possibility to detect, if present, dynamics with slower insurgence. Leon-Carrion et al. ${ }^{75}$ affirmed that highly activating stimulation can cause effects lasting long after stimuli cessation and, during the post-task can produce changes in meta- 
bolic indices even larger than those risen during the task period. As far as we know, our finding is the first one attesting a task-related cerebral hemodynamic change after the stimuli cessation. Based on the present results, we cannot resolve whether the $\mathrm{O}_{2} \mathrm{Hb}$ increase noticed in the post-task can be interpreted as an uncoupling between metabolic rate and flow changes, as a residual cortical activation, or rather as a new activation introduced by some sort of "alert" reaction due to the sudden ending of the task. In the first hypothesis, we associate the $\mathrm{O}_{2} \mathrm{Hb}$ decrease (observed in the last part of the active task) with a decrease in blood flow but not in metabolic rate (hemodynamic recoupling). In this perspective, interruption of stimulation should have effect mainly on the metabolic rate and not on the already diminished flow rate. This could origin an exceeding amount of $\mathrm{O}_{2} \mathrm{Hb}$ detected by the fNIRS as post-task activation. This overshoot could then be seen as a second uncoupling of the hemodynamic parameters, the first is caused by an increased CBF with smaller increase in $\mathrm{CMRO}_{2}$, the second is due to a decrease in $\mathrm{CMRO}_{2}$. Both mechanisms lead to an augmented $\mathrm{O}_{2} \mathrm{Hb}$ signal. A successive recoupling of blood flow and oxygen consumption perhaps brings about the final recovery to the basal level. This issue should anyway be addressed in further studies in order to better characterize the metabolic implications.

Since the cognitive stimulation lasted long time, a systemic increase in blood flow cannot be excluded. Further work is needed in order to investigate the effects of cognitive effort on systemic regulation in the brain. At this purpose, a comparison between hemodynamic changes measured using NIRS on the forehead and blood flow changes in carotid arteries measured by doppler sonography could be especially suitable and clarifying.

\section{CONCLUSIONS}

A characterization of cerebral functional response and its hemodynamic and metabolic correlates in the context of cognitive tasks is far to be fully understood. In this study, the duration and type of stimuli have been proven to greatly influence the hemodynamic and metabolic response. Prolonged stimulation of frontal areas deputed to attention and response inhibition have showed a long rise time $(3 / 4 \mathrm{~min})$ and no plateau, followed by a decrease in metabolic parameters toward a new steady state (recoupling) without significant performance drop. The end of the task highlighted either a task termination arousal or a hemodynamic/metabolic uncoupling, characterized by a second overshoot of $\mathrm{O}_{2} \mathrm{Hb}$ possibly indicating either an hemodynamic response at attention release or a persistence of $\mathrm{CBF}$ at a higher level than baseline and a concomitant decrease in $\mathrm{CMRO}_{2}$ consequent to the task end. The present finding demonstrates the capability of time-resolved fNIRS to monitor nontrivial functional dynamics during sustained attention tasks and their recovery. These results in healthy volunteers foster further investigations in clinical populations presenting attention impairment (e.g., post-traumatic patients) in order to monitor the functional correlates with routine attention tasks.

\section{ACKNOWLEDGMENTS}

The research leading to these results has received funding from the European Community's Seventh Framework Programme under the nEUROPt Project (Grant No. FP7HEALTH-2007-201076).

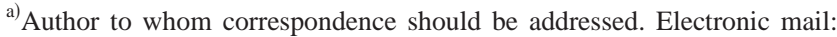
annamaria.bianchi@polimi.it; Present address: via Golgi 39, Campus Leonardo, Edificio 34, Quarto Piano, 20133 Milano, Italy; Telephone: 0039-02-2399-3342; Fax: 0039-02-23993360.

${ }^{1}$ P. J. Kirkpatrick, P. Smielewski, P. Al-Rawi, and M. Czosnyka, "Resolving extra-and intracranial signal changes during adult near infrared spectroscopy," Neurol. Res. 20, 19-22 (1998).

${ }^{2}$ J. M. Lam, P. Smielewski, P. Al-Rawi, P. Griffiths, J. D. Pickard, and P. J. Kirkpatrick, "Internal and external carotid contributions to near-infrared spectroscopy during carotid endarterectomy," Stroke 28(5), 906-911 (1997).

${ }^{3}$ A. Liebert, H. Wabnitz, J. Steinbrink, M. Möller, R. Macdonald, H. Rinneberg, A. Villringer, and H. Obrig, "Bed-side assessment of cerebral perfusion in stroke patients based on optical monitoring of a dye bolus by time-resolved diffuse reflectance," Neuroimage 24(2), 426-435 (2005).

${ }^{4}$ M. Wolf, M. Ferrari, and V. Quaresima, "Progress of near-infrared spectroscopy and topography for brain and muscle clinical applications," J. Biomed. Opt. 12(6), 062104 (2007).

${ }^{5}$ T. Ernst and J. Hennig, "Observation of a fast response in functional MR,” Magn. Reson. Med. 32(1, 146-149 (1994).

${ }^{6}$ K. C. Roberts, T. T. Tran, A. W. Song, and M. G. Woldorff, "Component structure of event-related fMRI responses in the different neurovascular compartments," Magn. Reson. Imaging 25(3), 328-334 (2007).

${ }^{7} \mathrm{E}$. Yacoub and X. Hu, "Detection of the early decrease in fMRI signal in the motor area," Magn. Reson. Med. 45, 184-190 (2001).

${ }^{8}$ P. A. Bandettini, E. C. Wong, R. S. Hinks, R. S. Tikofsky, and J. S. Hyde, "Time course EPI of human brain function during task activation," Magn. Reson. Med. 25, 390-397 (1992).

${ }^{9}$ R. B. Buxton, K. Uludağ, D. J. Dubowitz, and T. T. Liu, "Modeling the hemodynamic response to brain activation," Neuroimage 23, S220-S233 (2004).

${ }^{10}$ V. Toronov, A. Webb, J. H. Choi, M. Wolf, A. Michalos, E. Gratton, and D. Hueber, "Investigation of human brain hemodynamics by simultaneous near-infrared spectroscopy and functional magnetic resonance imaging," Med. Phys. 28(4), 521-527 (2001).

${ }^{11}$ H. Obrig, C. Hirth, J. G. Junge-Hülsing, C. Döge, R. Wenzel, T. Wolf, U. Dirnagl, and A. Villringer, "Length of resting period between stimulation cycles modulates hemodynamic response to a motor stimulus," Adv. Exp. Med. Biol. 411, 471-480 (1997).

${ }^{12}$ P. A. Bandettini, K. K. Kwong, T. L. Davis, R. B. Tootell, E. C. Wong, P. T. Fox, J. W. Belliveau, R. M. Weisskoff, and B. R. Rosen, "Characterization of cerebral blood oxygenation and flow changes during prolonged brain activation," Hum. Brain Mapp 5(2), 93-109 (1997).

${ }^{13}$ A. M. Howseman, D. A. Porter, C. Hutton, O. Josephs, and R. Turner, "Blood oxygenation level dependent signal time courses during prolonged visual stimulation,” Magn. Reson. Imaging 16(1), 1-11 (1998).

${ }^{14}$ H. R. Heekeren, H. Obrig, R. Wenzel, K. Eberle, J. Ruben, K. Villringer, R. Kurth, and A. Villringer, "Cerebral haemoglobin oxygenation during sustained visual stimulation-A near-infrared spectroscopy study," Philos. Trans. R. Soc. London, Ser. B 352(1354), 743-750 (1997).

${ }^{15}$ G. Matsuda and K. Hiraki, "Sustained decrease in oxygenated hemoglobin during video games in the dorsal prefrontal cortex: A NIRS study of children," Neuroimage 29, 706-711 (2006).

${ }^{16}$ F. Zhao, T. Jin, P. Wang, and S. G. Kim, "Improved spatial localization of post-stimulus BOLD undershoot relative to positive BOLD," Neuroimage 34(3), 1084-1092 (2007).

${ }^{17} \mathrm{M}$. Burke and C. Bührle, "BOLD response during uncoupling of neuronal activity and CBF," Neuroimage 32(1), 1-8 (2006).

${ }^{18}$ P. Marklund, P. Fransson, R. Cabeza, K. M. Petersson, M. Ingvar, and L. Nyberg, "Sustained and transient neural modulations in prefrontal cortex related to declarative long-term memory, working memory, and attention," Cortex 43(1), 22-37 (2007).

${ }^{19}$ G. Deutsch, A. C. Papanicolaou, W. T. Bourbon, and H. M. Eisenberg, "Cerebral blood flow evidence of right frontal activation in attention demanding tasks," Int. J. Neurosci. 36(1-2), 23-28 (1987). 
${ }^{20}$ D. Contini, A. Torricelli, A. Pifferi, L. Spinelli, F. Paglia, and R. Cubeddu, "Multichannel time-resolved system for functional near infrared spectroscopy," Opt. Express 14, 5418-5432 (2006).

${ }^{21}$ Y. Nomura, O. Hazeki, and M. Tamura, "Relationship between timeresolved and non-time-resolved Beer-Lambert law in turbid media," Phys. Med. Biol. 42, 1009-1023 (1997).

${ }^{22}$ D. Contini, A. Torricelli, A. Pifferi, L. Spinelli, and R. Cubeddu, "Novel method for depth-resolved brain functional imaging by time-domain NIRS," Proc. SPIE 6629, 662908 (2007).

${ }^{23}$ J. Steinbrink, H. Wabnitz, H. Obrig, A. Villringer, and H. Rinneberg, "Determining changes in NIR absorption using a layered model of the human head," Phys. Med. Biol. 46, 879-896 (2001).

${ }^{24}$ S. Del Bianco, F. Martelli, and G. Zaccanti, "Penetration depth of light re-emitted by a diffusive medium: Theoretical and experimental investigation," Phys. Med. Biol. 47, 4131-4144 (2002).

${ }^{25}$ R. A. Johnson and D. W. Wichern, Applied Multivariate Statistical Analysis, 5th ed. (Prentice-Hall, Upper Saddle River, NJ, 2002).

${ }^{26}$ K. J. Friston, "Statistical parametric mapping," in Functional Neuroimaging, edited by R. W. Thatcher, M. Hallet, T. Zeffiro, E. R. John, and M. Huerta (Academic, San Diego, 1994), pp. 79-93.

${ }^{27}$ K. J. Friston, A. P. Holmes, K. J. Worsley, J. P. Poline, C. D. Frith, and R. S. J. Frackowiak, "Statistical parametric maps in functional imaging: A general linear approach," Hum. Brain Mapp 2(4), 189-210 (1995).

${ }^{28} \mathrm{~K}$. J. Worsley and K. J. Friston, "Analysis of fMRI time-series revisitedagain," Neuroimage 2(3), 173-181 (1995).

${ }^{29}$ D. A. Harville, "Maximum likelihood approaches to variance component estimation and to related problems," J. Am. Stat. Assoc. 72, 320-338 (1997).

${ }^{30}$ G. D. Greenberg, Neuropsychological History Questionnaire (IDS, 1994).

${ }^{31}$ C. K. Conners, The Conners Continuous Performance Test, Multi-Health Systems, Toronto, Canada, 1994.

${ }^{32}$ J. C. Ballard, "Assessing attention: Comparison of response-inhibition and traditional continuous performance tests," J. Clin. Exp. Neuropsychol 23(3), 331-350 (2001).

${ }^{33}$ A. J. Fallgatter and W. K. Strik, "Right frontal activation during the continuous performance test assessed with near-infrared spectroscopy in healthy subjects," Neurosci. Lett. 223(2), 89-92 (1997)

${ }^{34}$ M. Okamoto, H. Dan, K. Sakamoto, K. Takeo, K. Shimizu, S. Kohno, I. Oda, S. Isobe, T. Suzuki, K. Kohyama, and I. Dan, "Three-dimensional probabilistic anatomical cranio-cerebral correlation via the international 10-20 system oriented for transcranial functional brain mapping," Neuroimage 21, 99-111 (2004).

${ }^{35}$ A. Torricelli, A. Pifferi, L. Spinelli, R. Cubeddu, F. Martelli, S. Del Bianco, and G. Zaccanti, "Time-resolved reflectance at null source-detector separation: improving contrast and resolution in diffuse optical imaging," Phys. Rev. Lett. 95, 078101 (2005).

${ }^{36}$ A. Pifferi, A. Torricelli, L. Spinelli, D. Contini, R. Cubeddu, F. Martelli, G. Zaccanti, A. Tosi, A. Dalla Mora, F. Zappa, and S. Cova, "Timeresolved diffuse reflectance using small source-detector separation and fast single-photon gating," Phys. Rev. Lett. 100, 138101 (2008)

${ }^{37}$ M. Kameyama, M. Fukuda, T. Uehara, and M. Mikuni, "Sex and age dependencies of cerebral blood volume changes during cognitive activation: A multichannel near-infrared spectroscopy study," Neuroimage 22(4), 1715-1721 (2004).

${ }^{38} \mathrm{P}$. Weber, J. Lutschg, and H. Fahnenstich, "Attention-induced frontal brain activation measured by near-infrared spectroscopy," Pediatr. Neurol. 31(2), 96-100 (2004)

${ }^{39}$ M. Toichi, R. L. Findling, Y. Kubota, J. R. Calabrese, M. Wiznitzer, N. K. McNamara, and K. Yamamoto, "Hemodynamic differences in the activation of the prefrontal cortex: Attention vs. higher cognitive processing," Neuropsychologia 42(5), 698-706 (2004).

${ }^{40}$ M. L. Schroeter, M. M. Bucheler, K. Muller, K. Uludag, H. Obrig, G. Lohmann, M. Tittgemeyer, A. Villringer, and D. Y. von Cramon, "Towards a standard analysis for functional near-infrared imaging," Neuroimage 21(1), 283-290 (2004)

${ }^{41}$ D. J. Mehagnoul-Schipper, B. F. van der Kallen, W. N. Colier, M. C. van der Sluijs, L. J. van Erning, H. O. Thijssen, B. Oeseburg, W. H. Hoefnagels, and R. W. Jansen, "Simultaneous measurements of cerebral oxygenation changes during brain activation by near-infrared spectroscopy and functional magnetic resonance imaging in healthy young and elderly subjects," Hum. Brain Mapp 16(1), 14-23 (2002).

${ }^{42}$ G. Strangman, J. P. Culver, J. H. Thompson, and D. A. Boas, "A quantitative comparison of simultaneous BOLD fMRI and NIRS recordings during functional brain activation," Neuroimage 17(2), 719-731 (2002). ${ }^{43}$ V. Toronov, S. Walker, R. Gupta, J. H. Choi, E. Gratton, D. Hueber, and A. Webb, "The roles of changes in deoxyhemoglobin concentration and regional cerebral blood volume in the fMRI BOLD signal," Neuroimage 19(4), 1521-1531 (2003).

${ }^{44}$ I. K. Christoffels, E. Formisano, and N. O. Schiller, "Neural correlates of verbal feedback processing: An fMRI study employing overt speech," Hum. Brain Mapp 28(9), 868-879 (2007).

${ }^{45}$ F. Hager, H. P. Volz, C. Gaser, H. J. Mentzel, W. A. Kaiser, and H. Sauer, "Challenging the anterior attentional system with a continuous performance task: A functional magnetic resonance imaging approach," Eur. Arch. Psychiatry Clin. Neurosci. 248(4), 161-170 (1998).

${ }^{46}$ M. M. Plichta, S. Heinzel, A. C. Ehlis, P. Pauli, and A. J. Fallgatter, "Model-based analysis of rapid event-related functional near-infrared spectroscopy (NIRS) data: A parametric validation study," Neuroimage 35(2), 625-634 (2007).

${ }^{47}$ J. Cohen-Adad, S. Chapuisat, J. Doyon, S. Rossignol, J. M. Lina, H. Benali, and F. Lesage, "Activation detection in diffuse optical imaging by means of the general linear model," Med. Image Anal. 11, 616-629 (2007).

${ }^{48}$ P. H. Koh, D. E. Glaser, G. Flandin, S. Kiebel, B. Butterworth, A. Maki, D. T. Delpy, and C. E. Elwell, "Functional optical signal analysis: A software tool for near-infrared spectroscopy data processing incorporating statistical parametric mapping," J. Biomed. Opt. 12(6), 064010 (2007).

${ }^{49}$ D. R. Leff, C. E. Elwell, F. Orihuela-Espina, L. Atallah, D. T. Delpy, A. W. Darzi, and G. Z. Yang, "Changes in prefrontal cortical behaviour depend upon familiarity on a bimanual co-ordination task: An fNIRS study," Neuroimage 39(2008), 805-813 (2007).

${ }^{50}$ C. S. Carter, T. S. Braver, D. M. Barch, M. M. Botvinick, D. Noll, and J. D. Cohen, "Anterior cingulate cortex, error detection, and the online monitoring of performance," Science 280(5364), 747-749 (1998).

${ }^{51}$ T. S. Braver, D. M. Barch, J. R. Gray, D. L. Molfese, and A. Snyder, "Anterior cingulate cortex and response conflict: Effects of frequency, inhibition and errors," Cereb. Cortex 11(9), 825-836 (2001).

${ }^{52}$ G. I. de Zubicaray, C. Andrew, F. O. Zelaya, S. C. R. Williams, and C. Dumanoir, "Motor response suppression and the prepotent tendency to respond: A parametric fMRI study," Neuropsychologia 38(9), 1280-1291 (2000).

${ }^{53}$ A. F. Mirsky, B. J. Anthony, C. C. Duncan, M. B. Ahearn, and S. G. Kellam, "Analysis of the elements of attention: A neuropsychological approach," Neuropsychol Rev. 2(2), 109-145 (1991).

${ }^{54}$ S. Konishi, K. Nakajima, I. Uchida, H. Kikyo, M. Kameyama, and Y. Miyashita, "Common inhibitory mechanism in human inferior prefrontal cortex revealed by event-related functional MRI," Brain 122, 981-991 (1999).

${ }^{55}$ J. V. Pardo, P. T. Fox, and M. E. Raichle, "Localization of a human system for sustained attention by positron emission tomography," Nature (London) 349(6304), 61-64 (1991).

${ }^{56} \mathrm{M}$. Sarter, B. Givens, and J. P. Bruno, "The cognitive neuroscience of sustained attention: Where top-down meets bottom-up,” Brain Res. Rev. 35(2), 146-160 (2001).

${ }^{57}$ E. Molteni, M. Butti, A. M. Bianchi, and G. Reni, "Activation of the prefrontal cortex during a visual $n$ - back working memory task with varying memory load: A near infrared spectroscopy study," Conference Proceedings of IEEE Engineering Medicine and Biology Society, 2008 (IEEE, 2008), pp. 4024-4027.

${ }^{58}$ M. L. Schroeter, S. Zysset, T. Kupka, F. Kruggel, and D. Yves von Cramon, "Near-infrared spectroscopy can detect brain activity during a colorword matching Stroop task in an event-related design," Hum. Brain Mapp 17(1), 61-71 (2002).

${ }^{59}$ M. J. Herrmann, M. M. Plichta, A. C. Ehlis, and A. J. Fallgatter, "Optical topography during a Go-NoGo task assessed with multi-channel nearinfrared spectroscopy," Behav. Brain Res. 160(1), 135-140 (2005).

${ }^{60}$ B. J. Casey, R. J. Trainor, J. L. Orendi, A. B. Schubert, L. E. Nystrom, J. N. Giedd, F. X. Castellanos, J. V. Haxby, D. C. Noll, J. D. Cohen, S. D. Forman, R. E. Dahl, and J. L. Rapoport, "A developmental functional MRI study of prefrontal activation during performance of a Go-No-Go task,” J. Cogn Neurosci. 9(6), 835-847 (1997).

${ }^{61}$ C. M. Adler, K. W. Sax, S. K. Holland, V. Schmithorst, L. Rosenberg, and S. M. Strakowski, "Changes in neuronal activation with increasing attention demand in healthy volunteers: An fMRI study," Synapse 42(4), 266$272(2001)$ 
${ }^{62}$ S. Ogawa, T. M. Lee, A. R. Kay, and D. W. Tank, "Brain magnetic resonance imaging with contrast dependent on blood oxygenation," Proc. Natl. Acad. Sci. U.S.A. 87(24), 9868-9872 (1990).

${ }^{63}$ F. Di Salle, E. Formisano, D. E. Linden, R. Goebel, S. Bonavita, A. Pepino, F. Smaltino, and G. Tedeschi, "Exploring brain function with magnetic resonance imaging," Eur. J. Radiol. 30(2), 84-94 (1999).

${ }^{64}$ S. G. Kim and K. Ugurbil, "Functional magnetic resonance imaging of the human brain," J. Neurosci. Methods 74(2), 229-243 (1997).

${ }^{65}$ R. Sharma and A. Sharma, "Physiological basis and image processing in functional magnetic resonance imaging: Neuronal and motor activity in brain," Biomed. Eng. Online 3(1), 13 (2004).

${ }^{66}$ G. M. Hathout, K. A. Kirlew, G. J. So, D. R. Hamilton, J. X. Zhang, U. Sinha, S. Sinha, J. Sayre, D. Gozal, R. M. Harper, and R. B. Lufkin, "MR imaging signal response to sustained stimulation in human visual cortex," Magn. Reson. Imaging 4(4), 537-543 (1994).

${ }^{67}$ J. Frahm, G. Krüger, K. D. Merboldt, and A. Kleinschmidt, "Dynamic uncoupling and recoupling of perfusion and oxidative metabolism during focal brain activation in man," Magn. Reson. Med. 35(2), 143-148 (1996).

${ }^{68}$ M. A. Mintun, B. N. Lundstrom, A. Z. Snyder, A. G. Vlassenko, G. L. Shulman, and M. E. Raichle, "Blood flow and oxygen delivery to human brain during functional activity: Theoretical modeling and experimental data," Proc. Natl. Acad. Sci. U.S.A. 98(12), 6859-6864 (2001).
${ }^{69} \mathrm{~K}$. Uludağ, "Transient and sustained BOLD responses to sustained visual stimulation," Magn. Reson. Imaging 26(7), 863-869 (2008).

${ }^{70}$ T. L. Luks, F. T. Sun, C. L. Dale, W. L. Miller, and G. V. Simpson, "Transient and sustained brain activity during anticipatory visuospatial attention," NeuroReport 19(2), 155-159 (2008).

${ }^{71}$ P. Hedera, D. Wu, J. S. Lewin, D. Miller, A. J. Lerner, and R. P. Friedland, "Temporal patterns of uncoupling between oxidative metabolism and regional cerebral blood flow demonstrated by functional magnetic resonance imaging," Invest. Radiol. 30(11), 625-633 (1995).

${ }^{72}$ G. Horn, "Neuronal mechanisms of habituation," Nature (London) 215(5102), 707-711 (1967).

${ }^{73}$ R. F. Thompson and W. A. Spencer, "Habituation: A model phenomenon for the study of neuronal substrates of behaviour," Psychol. Rev. 73, 16-43 (1966).

${ }^{74}$ Y. Hoshi, B. H. Tsou, V. A. Billock, M. Tanosaki, Y. Iguchi, M. Shimada, T. Shinba, Y. Yamada, and I. Oda, "Spatiotemporal characteristics of hemodynamic changes in the human lateral prefrontal cortex during working memory tasks," Neuroimage 20(3), 1493-1504 (2003).

${ }^{75}$ J. León-Carrión, J. F. Martín-Rodríguez, J. Damas-López, K. Pourrezai, K. Izzetoglu, J. M. Barroso y Martin, and M. R. Domínguez-Morales, “A lasting post-stimulus activation on dorsolateral prefrontal cortex is produced when processing valence and arousal in visual affective stimuli," Neurosci. Lett. 422(3), 147-152 (2007). 